\title{
Mothers' Postpartum Sleep Disturbance is Associated with the Ability to Sustain Sensitivity toward Infants
}

\author{
Lucy S. King ${ }^{1}$, Elizabeth Rangel ${ }^{2}$, Norah Simpson ${ }^{2}$, Liat Tikotzky ${ }^{3}$, and Rachel Manber ${ }^{2}$ \\ 1'Department of Psychology, Stanford University \\ ${ }^{2}$ Department of Psychiatry \& Behavioral Sciences, Stanford University \\ ${ }^{3}$ Department of Psychology, Ben-Gurion University of the Negev, Israel
}

\section{Corresponding author:}

Lucy S. King

Department of Psychology, Stanford University

Jordan Hall, 450 Serra Mall, Building 420

Stanford, CA 94305, USA

lucyking@stanford.edu 


\begin{abstract}
Infancy is a period of rapid development when the quality of caregiving behavior may be particularly consequential for children's long-term functioning. During this critical period for caregiving behavior, parents experience changes in their sleep that may affect their ability to provide sensitive care. The current study investigated the association of mothers' sleep disturbance with both levels and trajectories of maternal sensitivity during interactions with their infants. At 18 weeks postpartum, mothers and their infants were observed during a home-based ten-minute "free play" interaction. Mothers' nighttime sleep was objectively measured using actigraphy and subjectively measured using sleep diaries. Maternal sensitivity was coded in two-minute intervals in order to characterize changes in sensitivity across the free play interaction. We used exploratory factor analysis to reduce the dimensionality of the objective and subjective measures of mothers' sleep, identifying a subjective sleep disturbance and an objective sleep continuity factor. Using multi-level modeling, we found that mothers with poorer objective sleep continuity evidenced decreasing sensitivity toward their infants across the interaction. Mothers' self-reports of sleep disturbance were not associated with maternal sensitivity. Although future research is necessary to identify the mechanisms that may explain the observed association between poor sleep continuity and the inability to sustain sensitivity toward infants, mothers' postpartum sleep continuity may be one factor to consider when designing interventions to improve the quality of caregiving.
\end{abstract}

Keywords: postpartum sleep, maternal sensitivity, caregiving

Clinical Trial Registration: Clinicaltrials.gov, NCT01846585. 


\section{Introduction}

Infancy is a period of rapid development when caregiving behavior may be particularly consequential for children's long-term functioning. Maternal sensitivity, defined as the mother's ability to accurately perceive and interpret her infant's cues and respond to them appropriately and contingently (Ainsworth, Blehar, Waters, \& Wall, 1978), has been associated with a host of child outcomes, including infants' physiological responses to stress (Bosquet Enlow, King, et al., 2014), the establishment of secure attachment relationships (Wolff \& van IJzendoorn, 1997), children's ability to regulate negative affect (Davidov \& Grusec, 2006), and the development of internalizing symptoms (Kok et al., 2013) and externalizing problems (Belsky, Pasco Fearon, \& Bell, 2007). Further, infancy may be a critical period for the effects of the caregiving environment (McLaughlin et al., 2015; Smyke, Zeanah, Fox, Nelson, \& Guthrie, 2010), when parents' functioning has maximal impact on the long-term quality of the parent-child relationship and child wellbeing (Bagner, Pettit, Lewinsohn, \& Seeley, 2010; R. E. Perry, Blair, \& Sullivan, 2017).

During this critical period for caregiving behavior, parents experience changes in their sleep patterns (Gay, Lee, \& Lee, 2004). Young infants typically wake at least once during the night (Hysing et al., 2014; Price et al., 2014), increasing parents' wakefulness during their sleep period because of the need to take care of and feed their infant (Hunter, Rychnovsky, \& Yount, 2009; Mezick, 2013). Longitudinal studies employing actigraphy (i.e., wearable wrist accelerometry) measurements of sleep indicate that, whereas mothers' sleep duration does not deteriorate from the pre- to postpartum period, their sleep continuity, defined as wakefulness after sleep onset, significantly decreases (Montgomery-Downs, Insana, Clegg-Kraynok, \& Mancini, 2010). Following an initial postnatal drop in sleep continuity, mothers' sleep continuity improves across the first four months postpartum (Montgomery-Downs et al., 2010; Park, Meltzer-Brody, \& Stickgold, 2013); however, as many as $20-30 \%$ of all infants and toddlers have night-waking problems, and, accordingly, the sleep of many parents continues to be disturbed 
well beyond the first few months after birth (Mindell, Sadeh, Kwon, \& Goh, 2013; Piteo et al., 2013; Tikotzky et al., 2015; Volkovich, Bar-Kalifa, Meiri, \& Tikotzky, 2017).

Although few previous studies have examined the association between parents' sleep and their caregiving behavior, it is likely that parents' sleep disturbance during the postpartum periods impairs their ability to provide sensitive care. Indeed, in adults, sleep disturbance is associated with maladaptive functioning in multiple domains, including cognitive functioning, arousal level, affect regulation, and mood (Floam et al., 2015; Franzen, Siegle, \& Buysse, 2008; Mauss, Troy, \& LeBourgeois, 2013; Slama et al., 2017; Whitney et al., 2017). These effects are also apparent among postpartum women (Insana, Williams, \& Montgomery-Downs, 2013; Mcbean, Kinsey, Montgomery-downs, \& Virginia, 2016; McBean \& Montgomery-Downs, 2013; Swain, O'Hara, Starr, \& Gorman, 1997). In the broader literature on the antecedents of caregiving behavior, maladaptive functioning in each of these domains has been linked to less positive maternal behavior toward children (Crandall, Deater-Deckard, \& Riley, 2015; Leerkes, 2011; Sturge-Apple, Jones, \& Suor, 2017; Tester-Jones, Karl, Watkins, \& O’Mahen, 2016). Thus, postpartum sleep disturbance may have a negative impact on maternal sensitivity. A small number of studies have examined whether self-reported disturbance in mothers' sleep is related to mothers' reports of their feelings toward their infants, their parenting experiences, and their perceptions of their infants' behaviors. For example, mothers who reported spending more time awake at night caring for their infants reported lower levels of attachment to their infants (Tikotzky, 2016; Tikotzky, Chambers, Kent, Gaylor, \& Manber, 2012) and greater parenting stress (Di Blasio, Camisasca, \& Miragoli, 2018; Sinai \& Tikotzky, 2012) on questionnaires. Further, among mothers at risk for depression, those who reported waking more times during the night to care for their infants reported that their infants had more negative temperaments (Tikotzky, Chambers, Gaylor, \& Manber, 2010). Importantly, although mothers' self-reports of attachment to their infants, parenting stress, or perceptions of infant temperament may be correlated with caregiving behavior (Booth, Macdonald, \& Youssef, 2018; Muzik et al., 
2013; Perry, Dollar, Calkins, \& Bell, 2018), caregiving behavior was not directly measured in any of these prior studies. In fact, only two previous studies have directly examined the association between parents' sleep disturbance and caregiving behavior. Kim and Teti (2014) found no association between mothers' average self-reported sleep quality across the first six postpartum months and their bedtime emotional availability toward infants at age 9 months. Recently, however, Mcquillan, Bates, Staples, and Deater-Deckard (2019) found that actigraphy measures of mothers' sleep, including a composite measure reflecting wakefulness after sleep onset, were associated with less positive maternal bedtime caregiving behavior toward toddlers; in contrast, mothers' self-reported sleep problems were not associated with caregiving behavior. Further research is needed to clarify the associations of subjective and objective measures of maternal sleep disturbance with observed maternal sensitivity toward infants.

Maternal sensitivity is commonly measured through observations of maternal caregiving behavior during 5- to 15-minute laboratory-based parent-child interactions. Specifically, coders assign ratings of overall sensitivity that do not measure possible changes in sensitivity during the interaction period (e.g., Bigelow et al., 2010; Braungart-Rieker, Hill-Soderlund, \& Karrass, 2010; Thomas et al., 2017). A potentially important although under-investigated aspect of sensitive caregiving is the ability to sustain sensitivity over time. It is possible that some mothers may have the capacity to provide sensitive care for short intervals but struggle to maintain sensitivity across longer interactions with their infants. Although previous studies have found that maternal sensitivity decreases over time in the context of increasing infant distress during laboratory-based stressors (Bosquet Enlow, King, et al., 2014; Conradt \& Ablow, 2010; TesterJones et al., 2016), no study has previously investigated parents' ability to sustain sensitivity during naturalistic play interactions with their infants.

There are at least two reasons why maternal sensitivity may decrease across a parentchild interaction. First, demand characteristics and social desirability effects may lead mothers to engage in positive behavior that does not reflect their behavior when they are not being 
observed (Holden, Williamson, \& Holland, 2014; Lee et al., 2017); further, these factors may have larger impacts on mothers' behavior at the beginning of an interaction and wear off as mothers acclimate to the presence of the cameras or become distracted from the goal of attending to the infant. Indeed, distraction is one method used to reduce demand characteristics in the laboratory (McCambridge, de Bruin, \& Witton, 2012). Thus, maternal sensitivity during the latter part of an interaction may more accurately reflect children's everyday experiences during interactions with their caregivers. Alternatively, it is possible that it is challenging to sustain attention toward infants over time; in fact, it is well-documented that attention, and consequently performance, decreases over time during mundane tasks, a phenomenon known as the "vigilance decrement" (Thomson, Besner, \& Smilek, 2015). Given that an important antecedent of maternal sensitivity is the ability to attend to often subtle child cues (Leerkes, 2011), waning maternal attention across a parent-child interaction may adversely affect maternal sensitivity. Relevant to the current study, maintaining sensitivity over time may be particularly challenging for mothers experiencing poor sleep continuity during the postpartum period. Indeed, in a longitudinal study in which mothers completed the psychomotor vigilance test (PVT, a measure of sustained attention) each morning across the first 12 weeks postpartum, more fragmented maternal sleep was associated with poorer performance on the PVT (Insana et al., 2013).

The current study addresses critical gaps in our knowledge regarding the association between mothers' sleep disturbance and caregiving behavior during the postpartum period. More specifically, the goal of the current study was to use data collected in a randomized controlled trial of treatment for perinatal insomnia (Manber et al., 2019) to investigate the association between mothers' sleep disturbance in the postpartum period and observed sensitivity during mother-infant interactions at home. Given that sleep is a multi-dimensional construct that cannot be fully characterized using a single measure or method (Buysse, 2014), we used a multi-dimensional and multi-method approach to measure mothers' sleep disturbance, including indicators of nighttime sleep disturbance using both self-report sleep 
diaries and objective actigraphy measurements. We conducted an exploratory factor analysis in order to reduce the dimensionality of these measures. In this secondary analysis of data from the parent RCT, we hypothesized that mothers with more disturbed sleep would evidence lower overall levels of sensitivity during a ten-minute "free play" interaction with their infants, and, further, would evidence greater difficulty sustaining higher levels of sensitivity across this interaction.

\section{Methods}

\subsection{Participants}

The parent study for the current analyses enrolled 194 pregnant women with insomnia disorder to participate in a randomized controlled trial $(\mathrm{RCT})$ of treatment for perinatal insomnia. In this RCT (Manber et al., 2019), women with insomnia disorder (American Psychiatric Association, 2013) were randomized to receive either cognitive behavioral therapy for insomnia (CBTi) or an active control insomnia therapy, with five treatment sessions provided during pregnancy and a sixth session at six weeks postpartum. The sample for the current analyses included women who participated in a follow-up home visit at 18 weeks postpartum. At this visit, mother-infant dyads participated in a video-recorded free play interaction for the assessment of maternal sensitivity (described below). Of the original 194 women enrolled in the study, 94 attended the 18-week follow-up visit. Of these 94 women, 17 did not attempt to complete the video-recorded interaction (were unwilling to be video-recorded or could not complete the full protocol due to scheduling difficulties or illness). Of the 77 who attempted to complete the interaction, 67 provided usable data (4 dyads did not complete because infant was crying, sick, or sleepy and data from 6 dyads were lost due to video-recording errors). Therefore, the final analyzable sample for the current study included the 67 mother-infant dyads who successfully completed the interaction.

We present demographic and clinical characteristics for the final sample of 67 motherinfant dyads in Table 1. We compared the baseline demographic and clinical characteristics of 
these 67 women to the $127 / 194$ women who were enrolled but who were not included in the analyzable sample. Compared to these 127 women, women in the final sample were less likely to identify as Latina ( $28 \%$ vs $43 \%$ ), and were more likely to identify as racially White ( $54 \%$ vs $43 \%)$. 
Table 1. Descriptive statistics of the study sample

\begin{tabular}{|c|c|c|}
\hline & $\begin{array}{l}\text { Mean (SD) } \\
\text { or N }\end{array}$ & Range \\
\hline Mother's age (years) & $34.58(5.82)$ & $23.78-48.62$ \\
\hline Infant's age (months) & $4.27(0.23)$ & $3.84-5.10$ \\
\hline Maternal sensitivity & $3.71(0.92)$ & $1.70-5.90$ \\
\hline $\begin{array}{l}\text { Infant negative mood } \\
\text { Objective maternal sleep }\end{array}$ & $1.79(0.86)$ & $1.00-4.25$ \\
\hline WASO (minutes) & 57.35 (26.39) & $17.68-138.50$ \\
\hline Arousals ( $n$ ) & $18.29(5.05)$ & 9.00-31.00 \\
\hline TST (minutes) & 420.43 (70.95) & $238.67-640.60$ \\
\hline \multicolumn{3}{|l|}{ Subjective maternal sleep } \\
\hline WASO (minutes) & 36.92 (28.59) & $0.00-117.86$ \\
\hline Awakenings ( $n$ ) & $2.03(1.19)$ & $0.00-5.00$ \\
\hline TST (minutes) & 412.19 (70.97) & $184.20-540.00$ \\
\hline Quality (Likert scale) & $3.18(0.74)$ & $1.71-4.86$ \\
\hline Insomnia severity & 7.17 (5.19) & $0-20$ \\
\hline Depressive symptoms & $5.18(4.43)$ & $0-15$ \\
\hline Latina & 19 & \\
\hline \multicolumn{3}{|l|}{ Race } \\
\hline White & 36 & \\
\hline Black & 1 & \\
\hline Asian: India & 2 & \\
\hline Asian: Other countries & 11 & \\
\hline \multirow{2}{*}{\multicolumn{3}{|c|}{ Alaskan Native }} \\
\hline & & \\
\hline $\begin{array}{l}\text { Native Hawaiian/Pacific } \\
\text { Islander }\end{array}$ & 0 & \\
\hline Mixed Race & 5 & \\
\hline Other & 9 & \\
\hline Unable to answer & 2 & \\
\hline \multicolumn{3}{|l|}{ Income $^{2}$} \\
\hline$<\$ 14,999$ & 4 & \\
\hline$\$ 15,000-29,999$ & 14 & \\
\hline$\$ 30,000-54,999$ & 2 & \\
\hline$\$ 55,000-99,999$ & 9 & \\
\hline$\geq \$ 100,000$ & 34 & \\
\hline Unable to answer & 4 & \\
\hline
\end{tabular}

Notes. Maternal sensitivity is the average across the five two-minute intervals of the free play interaction. "Subjective" maternal sleep measures are mothers' reports on sleep diaries: WASO is the wake time after sleep onset during nighttime sleep due to caregiving for the infant; Awakenings is the number of awakenings to care for the infant during the night; TST is total nighttime sleep time (duration). "Objective" sleep measures are actigraphy measures: WASO is wake time after sleep onset during nighttime sleep; arousals is the number of wakeful periods after sleep onset $\geq 1$ minute; TST is total nighttime sleep. Although the modal reported income was $\geq \$ 100,000$, this value must be interpreted in reference to area cost of living. The lowincome limit for a family of four in Santa Clara County ranged from $\$ 75,050$ to $\$ 79,250$ during the data collection period (www.huduser.gov). 


\subsection{Procedure}

This study was approved by the Stanford University Institutional Review Board. Pregnant women were recruited from university-based obstetric clinics, county-hospital obstetric clinics, and through community advertising around the San Francisco Bay Area, California to participate in an RCT of treatment for perinatal insomnia. Inclusion criteria were that women were fluent in English or Spanish, were between 18-32 weeks gestation at the initial screening visit, and met criteria for insomnia disorder based on the Diagnostic and Statistical Manual for Mental Disorder - Fifth Edition (DSM-5; American Psychiatric Association, 2013) with minimum duration reduced from three to one month. Participants were excluded if they had an unstable medical condition or if they met diagnostic criteria for current major depressive disorder, current panic disorder with nocturnal panic attacks occurring more than four times in the past month, current posttraumatic stress disorder, substance abuse/dependence during pregnancy, or life time diagnosis of bipolar disorder or a thought disorder. At 18 weeks postpartum (+/- 2 weeks), participants were scheduled for an in-home study visit, during which mothers completed questionnaires; participated in the video-recorded mother-infant interaction; and wore actigraphs for monitoring of their sleep while concurrently completing sleep diaries (described below).

\subsection{Measures}

2.4.1 Maternal sensitivity. We measured maternal sensitivity using observational coding of a ten-minute "free play" mother-infant interaction conducted at the 18-week postpartum home visit. Interactions took place in areas of the home where mothers usually interacted with their infants and were video-recorded for subsequent coding. Dyads had access during the interaction to the toys they usually played with and could play in any position while remaining in view of the video camera. Home-based assessments of caregiving behavior may be more ecologically valid than laboratory-based assessments (Bernard, Meade, \& Dozier, 2013). 
We rated maternal sensitivity from the video-recordings using the infant adaptation of the Parent-Child Interaction Rating Scales ([PCIRS-IA]; Bosquet Enlow, Carter, Hails, King, \& Cabrera, 2014; Sosinsky, Marakovitz, \& Carter, 2004). Two trained independent coders (authors L.K. and E.R.) rated maternal sensitivity during each two-minute interval of the video-recorded free play period on a scale of 1 (very low) to 7 (very high) in half-point increments. Coding maternal sensitivity in repeated intervals allowed for examination of changes in sensitivity across the ten-minute free play interaction. The PCIRS-IA qualitatively defines maternal sensitivity as the mother "manifesting an awareness of the child's needs, moods, interests, and capabilities" and responding appropriately and contingently to infant cues (Sosinsky et al., 2004). In addition, given the potentially transactional nature of parent-infant interactions, we rated infant negative mood during the free play, defined by "the extent to which the child cries, whimpers, fusses, frowns, screams, tenses body while crying, or otherwise expresses his/her discontentment, anger or hostility" (Sosinsky et al., 2004). To ensure reliability and prevent coder drift, every fourth video was rated by both coders. If the two coders differed in their scores by $>1$ for any two-minute interval, they discussed and determined consensus scores. Reliability for maternal sensitivity at the level of 2-minute interval was good $(\mathrm{ICC}=.81)$ and reliability for infant negative mood was acceptable $(\mathrm{ICC}=.77)$.

2.4.2 Maternal sleep disturbance. We used a multi-dimensional and multi-method approach to characterize maternal sleep disturbance.

Objective sleep measures. Wrist actigraphy is a valid method (Marino et al., 2013) for objectively assessing sleep in the home environment over multiple nights based on data collected by a sensitive accelerometer embedded within the unit. In this study, we asked mothers to wear the Actiwatch $2^{\mathrm{TM}}$ on their non-dominant hand each night for at least 7 nights. For analyses that used actigraphy data, we excluded participants who did not have valid actigraphy data within two weeks of the 18-week home visit (i.e., they did not complete actigraphy, they completed actigraphy $>2$ weeks prior to the home visit, or there was a $>2$ week 
delay in completing it after the home visit); 55 mothers provided valid actigraphy data within this timeframe. On average, these participants completed 6.58 nights $(S D=1.34)$ of actigraphy recording.

Participants were asked to wear the Actiwatch unit on their non-dominant hand and press an event marker to indicate when they turned off the lights to attempt to fall asleep and again when they got out of bed in the morning. The actigraphy data were scored using the Philips Respironics Actiware software (version 6.0.9) in one minute epochs between lights out and wake time. A research assistant identified the scoring period based on a combination of three factors, which were rank ordered as follows: event markers, sleep diary, and marked changes in light and activity signals. If scored by light and activity changes, we defined the beginning of sleep by the beginning of the first immobility interval (as defined by the software scoring algorithm) after turning off the light that lasted at least 10 minutes. We calculated three summary sleep variables from the actigraphy data, averaging across nights of recording: 1) mean objective time awake after sleep onset (the number of minutes the mother spent awake after falling asleep and prior to getting up for the day [WASO]), 2) mean objective number of arousals (the number of wakeful periods after sleep onset that lasted at least one minute), and 3) objective total nighttime sleep duration (objective total sleep time [TST]).

Subjective sleep measures. Mothers completed the Consensus Sleep Diary (Carney et al., 2012) daily and concurrent with their actigraphy recordings. Following each night of sleep, mothers reported the amount of time they spent awake caring for the infant, how many times they woke up to take care of the infant, their total nighttime sleep duration, and the perceived quality of their sleep. We calculated four summary sleep variables from the sleep diary data, averaging across nights: mean subjective WASO, mean subjective number of awakenings, mean subjective TST, and mean perceived sleep quality measured on a scale ranging from 1very poor to 5-very good. For analyses that used sleep diary data, we excluded participants who did not have sleep diary data within two weeks of the 18-week home visit; 59 mothers provided 
valid sleep diary data within this timeframe. On average, these participants completed sleep diaries for 7 nights $(S D=1.45)$.

2.4.3 Maternal psychopathology symptoms. At the 18-week home visit, mothers reported their current symptoms of insomnia and depression.

Self-reported insomnia severity. Mothers completed the Insomnia Severity Index (ISI), a validated 7-item measure for the assessment of insomnia symptoms (Bastien, Vallie, \& Morin, 2001). Mothers reported their symptoms for two weeks prior to the home visit. In the current sample, the internal reliability for the ISI was good ( $\alpha=.87)$. One mother did not complete the ISI.

Maternal Depressive Symptoms. Mothers reported the severity of their depressive symptoms on the Edinburgh Postnatal Depression Scale (EPDS; Cox, Holden, \& Sagovsky, 1987). In the current sample, reliability for the EPDS was good ( $\alpha=.83)$. One mother did not complete the EPDS.

\subsection{Data analysis}

Analysis scripts are available at https://github.com/lucysking/sleep caregiving. Data are available upon request. We conducted analyses in R (R Core Team, 2018).

First, we conducted an exploratory factor analysis (EFA) in order to reduce the dimensionality of the data characterizing mothers' sleep disturbance. This data-driven approach reveals the covariance structure among the seven sleep measures, or, in other words, how these measures "go together". We implemented the EFA using the "fa" function in the "psych" package (Revelle, 2018), entering each of the seven sleep measures as indicator variables. We used Pearson correlations to find minimum residual solutions and varimax rotation to improve the interpretability of the factors (Revelle, 2018). We used a scree test to determine the number latent factors with eigen values $>1.0$ (Costello \& Osborne, 2005), and interpreted their meaning by examining the factor loadings. Based on the results of the EFA, we summed sleep measures that loaded highly onto the same factor, which is the recommended approach when risk factors 
are overlapping (Kraemer et al., 2001). We used these combined sleep measures in our primary analyses testing the hypothesis that mothers with more disturbed sleep evidence lower overall sensitivity and difficulty sustaining sensitivity over time. Importantly, by focusing on the combined rather than the individual sleep measures we reduced the number of tests conducted, decreasing the Type I error rate.

Given the nested structure of the data (multiple ratings of maternal sensitivity nested within individuals), we used separate multi-level models (also known as hierarchical linear or mixed-effects models [Woltman, Feldstain, Mackay, \& Rocchi, 2012]) to test associations between the combined sleep measures and both overall levels of maternal sensitivity and linear change (i.e., increases or decreases) in levels of maternal sensitivity across the observed free play period. We implemented these models using the function "Imer" in the "Ime4" package, and we used the package "ImerTest" for calculation of degrees of freedom and p-values (Bates, Machler, Bolker, \& Walker, 2014; Kuznetsova, Brockhoff, \& Christensen, 2016). We tested our hypothesis by specifying a main effect of the combined sleep measure and a free play interval * sleep measure interaction. Free play interval was treated continuously, coded as following: 0 (02 minutes], 2 (2-4 minutes), 4 (4-6 minutes), 6 (6-8) minutes, and 8 (8-10 minutes). In this model, a significant main effect of the sleep measure indicated that the measure was associated with overall levels of maternal sensitivity, whereas a significant interaction between free play interval and the sleep measure indicated that the measure was associated with linear change in sensitivity across the observed free play period.

We probed significant interactions by examining the simple slopes of free play interval at +/- 1SD from the mean of the sleep measure. Intervention condition and intervention site were included as covariates in all models. We effect-coded nominal variables (condition, site) and mean-centered all continuous variables except for free play interval, which was centered at 0 (i.e., the first interval). In secondary analyses, we tested whether the effects of sleep disturbance remained significant after adjusting for both the main and interactive effects 
(variable * free play interval) of maternal depressive symptom severity, family income, and infant negative mood during the observed free play period. In addition, we examined associations of self-reported symptoms of insomnia with maternal sensitivity.

\section{Results}

\subsection{Sample Characteristics}

We present characteristics of the study sample in Table 1, including the mean, standard deviation, and range for each of the seven sleep measures and the observational ratings of maternal sensitivity and infant negative mood. At the 18-week home visit, there were no significant differences in sleep variables, maternal sensitivity, or maternal race and ethnicity between those randomized to receive $\mathrm{CBTi}(\mathrm{n}=34)$ and those randomized to receive active control insomnia therapy during pregnancy $(n=33)$.

\subsection{Correlations among sleep measures}

We present a heatmap of the correlations among the seven sleep measures in Figure 1. Objective WASO and objective number of arousals were strongly positively correlated ( $r=.75$ ); however, objective TST was not associated with either objective WASO or objective number of arousals ( $r$-values<.16). All of the subjective measures were intercorrelated, such that subjective WASO was positively correlated with subjective number of awakenings ( $r=.53)$, and negatively correlated with subjective TST $(r=-.59)$ and perceived sleep quality $(r=-.46)$. Objective WASO and objective arousals were weakly correlated with subjective WASO and subjective number of awakenings ( $r$-values $<.30$ ), respectively. Objective TST was also weakly associated with subjective TST ( $r=.26)$ and unassociated with subjective WASO, awakenings, and perceived sleep quality ( $r$-values<.17). 
Figure 1. Heatmap of correlations among objective and subjective measures of mothers' sleep.

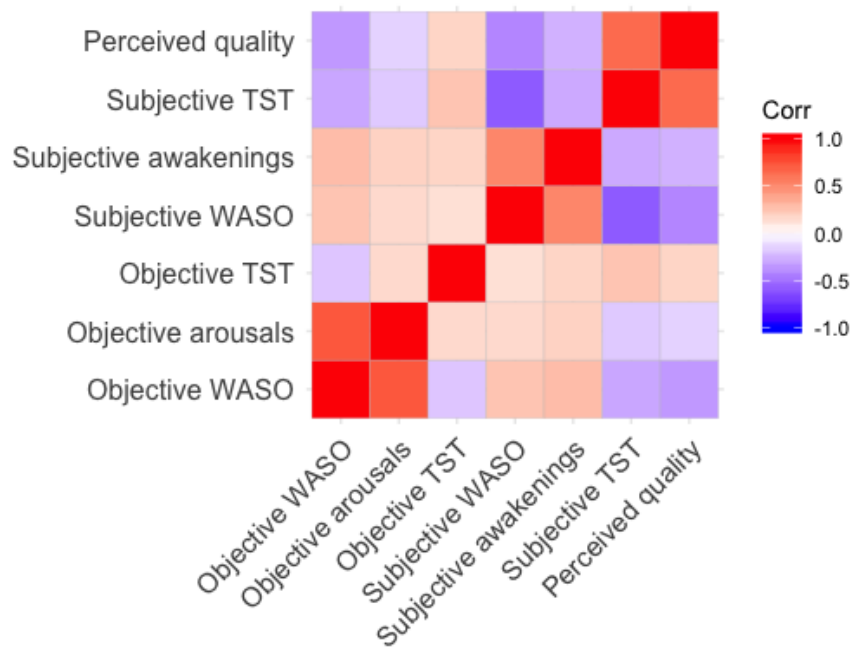

Notes. "Objective" measures are based on nightly actigraphy. "Subjective" measures are based on daily sleep diary responses. WASO = wake time after sleep onset. TST = total nighttime sleep time.

\subsection{Exploratory factor analysis}

Bartlett's test of sphericity indicated that the seven sleep measures were factorable $\left(\chi^{2}(21)=260.66, p<.001\right)$. A scree plot revealed three factors with eigen values $\geq 1$; however, the decline in eigen value was most pronounced between the second and third factors, indicating that two factors should be retained. In an initial test of a two-factor solution, objective TST loaded weakly onto both factors (loadings $<.16$ ); therefore, we dropped this variable from the factor analysis. In the final model, the two identified factors collectively explained $59 \%$ of the variance in the sleep data (factor 1: $33 \%$, factor $2: 26 \%$ ). We present a heatmap of the factor loadings in Figure 2. We labeled the first factor subjective sleep disturbance: whereas subjective WASO and awakenings had high positive loadings on this factor, subjective TST and perceived sleep quality had high negative loadings. We labeled the second factor objective sleep continuity: objective WASO and arousals had high positive loadings on this factor; none of the other measures loaded strongly onto this factor. 
Figure 2. Factor loadings of objective and subjective measures of mothers' sleep.

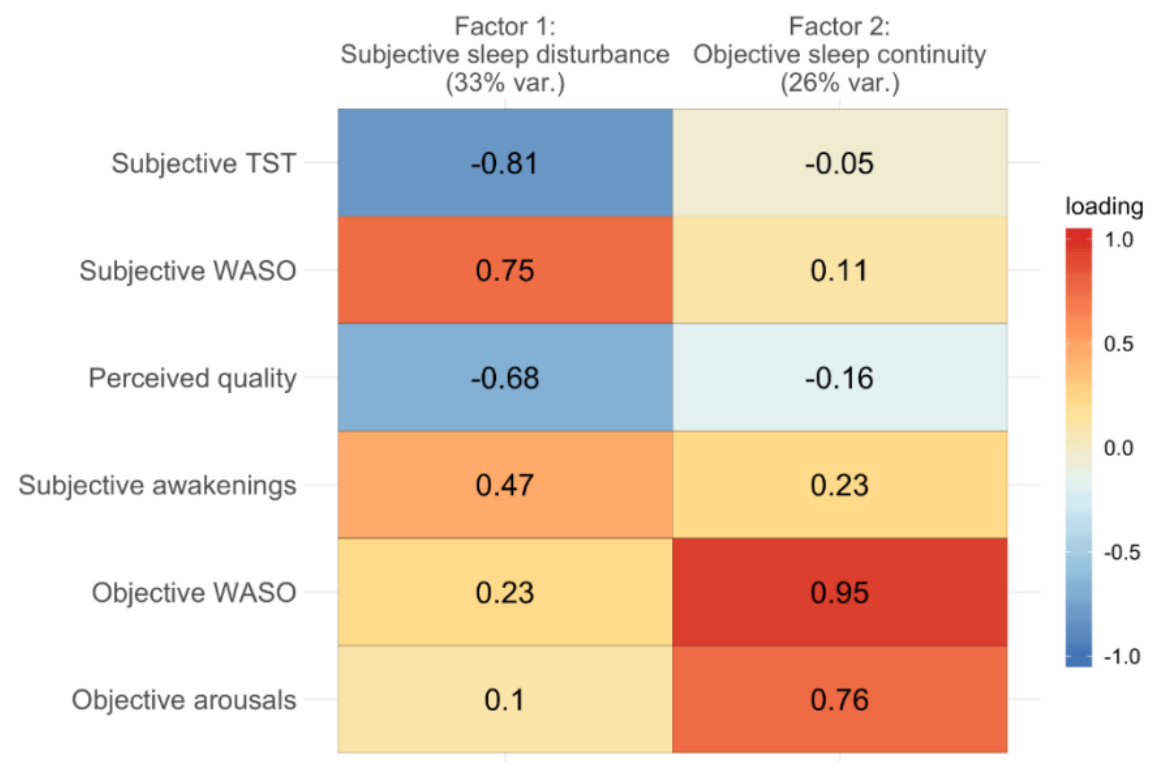

Notes. "Objective" measures are based on nightly actigraphy. "Subjective" measures are based on daily sleep diary responses about nighttime sleep. WASO = wake time after sleep onset. TST $=$ total nighttime sleep time. Factor 1 explained $33 \%$ of the variance and factor 2 explained $26 \%$ of the variance (var. = variance). Values in each cell are the factor loadings and the color bar indicates the strength of the loading in the positive or negative direction.

When multiple measures are correlated and likely to be co-dominant in predicting risk, combining them generally increases the reliability of the measure of the shared construct (Kraemer et al., 2001). Therefore, based on the results of the EFA, we combined the sleep measures to compute subjective sleep disturbance and objective sleep continuity summary scores derived from standardized scores for each measure (i.e., z-scores) as follows: to operationalize subjective sleep disturbance, we summed subjective WASO and subjective number of awakenings and then subtracted subjective TST and perceived sleep quality from this sum; to operationalize objective sleep continuity, we summed objective WASO and objective number of arousals. We then standardized the objective sleep disturbance and objective sleep continuity scores and multiplied objective sleep continuity scores by -1 . Thus, 
higher subjective sleep disturbance scores indicated more disturbed sleep and lower objective sleep continuity scores indicated worse continuity.

\subsection{Association of subjective sleep disturbance with maternal sensitivity}

We first tested the hypothesis that mothers with greater subjective sleep disturbance evidence lower overall levels of maternal sensitivity and decreases in sensitivity across the free play period when controlling for intervention condition and site. Results of a multi-level model regressing free play interval and an interaction between free play interval and subjective sleep disturbance on the repeated measure of maternal sensitivity indicated that there was no significant main effect of subjective sleep disturbance on overall maternal sensitivity $(\beta=0.02$, $\mathrm{SE}=0.13, t(71.19)=-0.12, p=.906)$, nor did free play interval and subjective sleep disturbance interact to explain changes in maternal sensitivity across the five two-minute free play intervals $(\beta=0.04, \mathrm{SE}=0.06, t(216.13)=-0.74, p=.460)$.

\subsection{Association of objective sleep continuity with maternal sensitivity}

We next tested the hypothesis that mothers with poorer objective sleep continuity evidence lower overall levels of maternal sensitivity and decreases in sensitivity across the free play interaction. Results of a multi-level model regressing free play interval and an interaction between free play interval and objective sleep continuity on the repeated measure of maternal sensitivity indicated that there was no significant main effect of objective sleep continuity on maternal sensitivity $(\beta=-0.01, \mathrm{SE}=0.13, t(74.79)=-0.14, p=.909)$; however, there was a significant interaction between objective sleep continuity and free play interval $(\beta=0.14$, $\mathrm{SE}=0.06, t(217.15)=2.32, p=.021,95 \% \mathrm{Cl}[0.02,0.25])$, indicating that objective sleep continuity was associated with changes in maternal sensitivity across the five two-minute free play intervals.

To interpret this interaction, we examined the simple slopes of free play interval at +/1SD from the mean of objective sleep continuity. Among mothers with better objective sleep 
continuity, maternal sensitivity did not diminish across the free play period $(\beta=-.01, \mathrm{SE}=0.13$, $t(217.34)=-0.23, p=.818)$; however, mothers with poorer objective sleep continuity demonstrated significant decreases in maternal sensitivity across the free play period $(\beta=-0.17, \mathrm{SE}=0.05$, $t(217.12)=-3.53, p=<.001,95 \% \mathrm{CI}[-0.26,-0.07])$. In Figure 3, we have plotted these estimated simple slopes at 1SD from the mean of objective sleep continuity (i.e., poorer continuity), the mean of sleep continuity, and +1 SD from the mean of sleep continuity (i.e., better sleep continuity).

Figure 3. Associations of mothers' objective sleep continuity with linear change in maternal sensitivity across the "free play" interaction

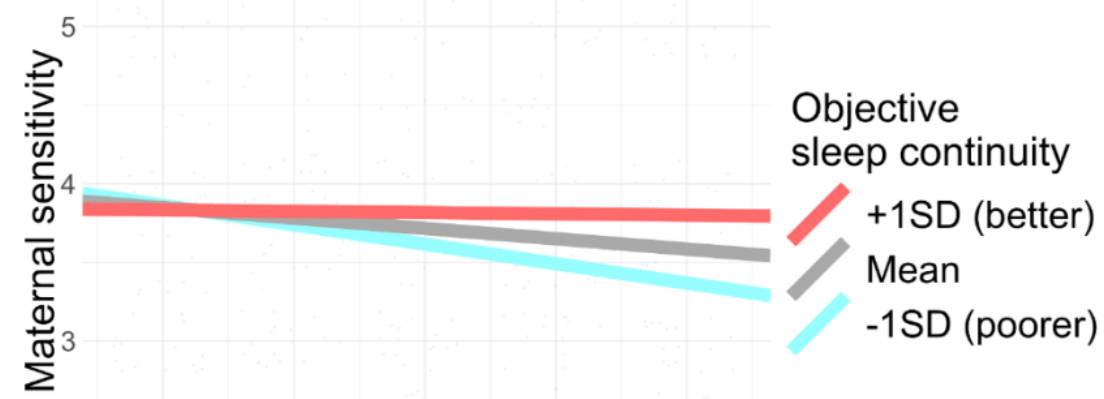

2

$$
{ }^{2} \text { Free play interval (minutes) }{ }^{6}
$$

Notes.: Mothers' objective sleep continuity is operationalized as a standardized sum of the actigraphy measures of WASO and number of arousals. Maternal sensitivity was observationally coded in two-minute intervals across the ten-minute "free play" interaction. Simple slopes are the estimated associations between free play interval and maternal sensitivity at +1 SD from the mean of sleep continuity (blue), the mean of sleep continuity (grey), and -1SD from the mean of sleep continuity (red). Poorer sleep continuity was associated with significant decreases in sensitivity across the interaction.

To facilitate clinically meaningful interpretability of the observed association between mothers' objective sleep continuity and linear change in maternal sensitivity across the free play 
period, we next conducted complementary analyses separately focused on objective WASO and objective arousals. Specifically, we conducted Johnson-Neyman region of significance tests using the "jtools" package in R (Long, 2018) to identify the values of WASO and arousals at which the trajectory of maternal sensitivity across the five two-minute intervals became significantly negative, Results of this test indicated that the trajectory of maternal sensitivity was significantly negative when mothers spent $\geq 52$ minutes awake after sleep onset (Figure 4) or experienced $\geq 17$ arousals. Fifty-three percent of mothers had mean objective WASO scores $\geq$ 52 minutes and $58 \%$ of mothers experienced a mean of $\geq 17$ arousals a night.

Figure 4. Johnson-Neyman region of significance test of the association of objective WASO with the trajectory of maternal sensitivity across the mother-infant interaction

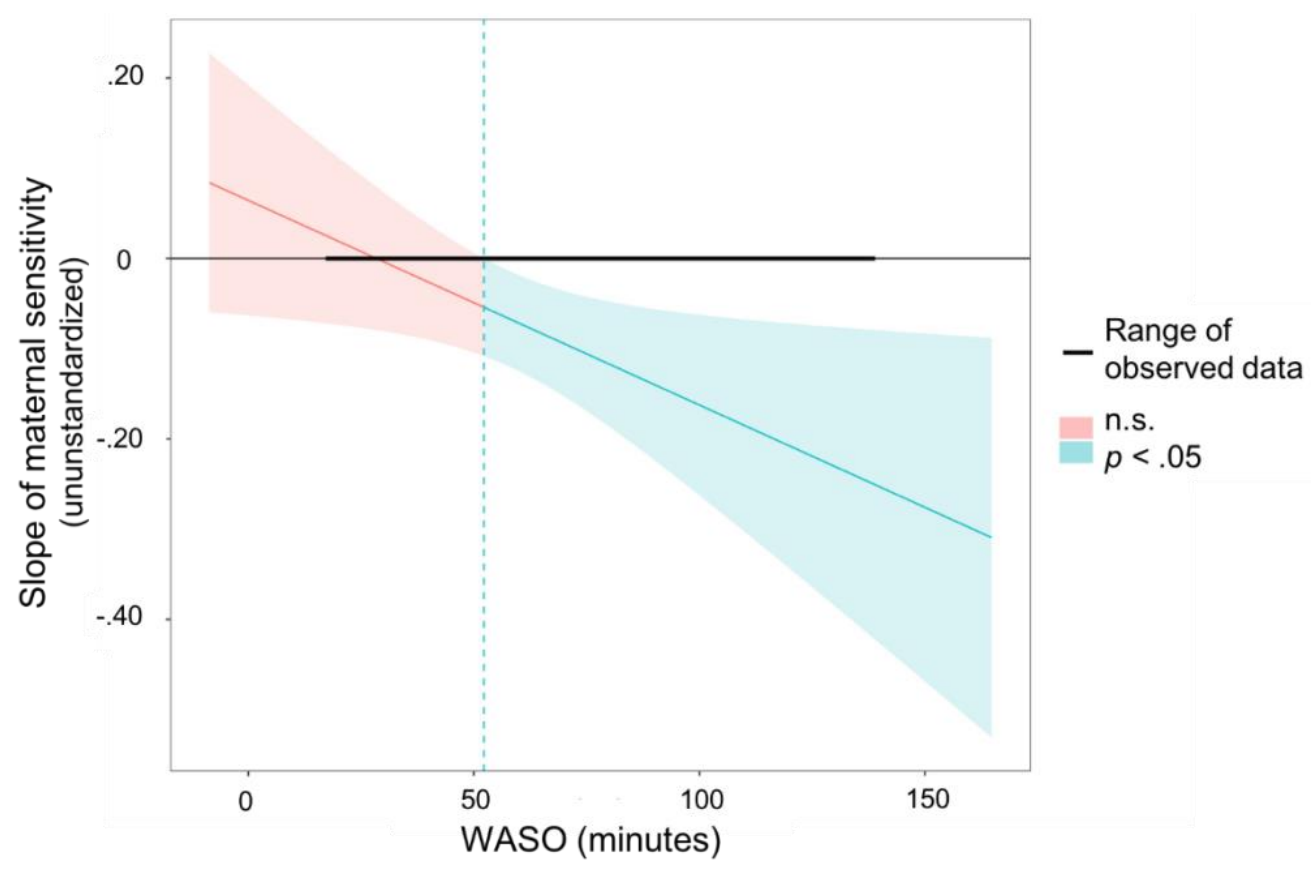

Notes. Slope of maternal sensitivity is the estimated slope of maternal sensitivity across the free play interaction. WASO is wake time after sleep onset measured by actigraphy. The JohnsonNeyman region of significance test provides the value of the moderator (WASO) at which the slope of the predictor (the effect of time during the free play interaction on maternal sensitivity) becomes significant at $\alpha=.05$. The trajectory of maternal sensitivity was significantly negative for mothers who spent approximately 52 or more minutes awake after sleep onset.

\subsection{Association of objective total sleep time with maternal sensitivity}


Given that objective TST was weakly correlated with the other measures of subjective and objective sleep, we secondarily tested its association with maternal sensitivity. Results of a multi-level model regressing free play interval and an interaction between free play interval and objective TST on the repeated measure of maternal sensitivity indicated that there was no significant main effect of objective TST on overall maternal sensitivity $(\beta=0.07, S E=0.13$, $t(73.75)=0.55, p=.583)$, nor did free play interval and objective TST interact to explain changes in maternal sensitivity across the five two-minute free play intervals $(\beta=-.29, S E=0.21$, $t(217.20)=-1.38, p=.179)$.

\subsection{Additional analyses}

We additionally evaluated whether the association between objective sleep continuity and the trajectory of maternal sensitivity remained significant when covarying maternal depressive symptom severity, infant negative mood, and household income. Specifically, we added the main and interactive (free play interval * variable) effects of depressive symptom severity, infant negative mood, and income to the original model as covariates. The interaction between objective sleep continuity and free play interval remained significant when adjusting for the effects of depressive symptom severity, infant negative mood, and income $(\beta=.16, S E=0.06$, $t(211.17)=-2.71, p=.007,95 \% \mathrm{Cl} 0.05,0.28])$. Finally, we examined whether mothers' symptoms of insomnia were associated with overall levels of maternal sensitivity or linear change in sensitivity across the free play. Insomnia severity was not associated with overall levels of maternal sensitivity $(\beta=-.14, \mathrm{SE}=0.12, t(88.11)=-1.18, p=.243)$, nor with the trajectory of maternal sensitivity across the free play period $(\beta=-.02, \mathrm{SE}=0.05, t(260.31)=-0.36, p=.718)$.

\section{Discussion}

In the first known study of the association between mothers' sleep disturbance during the postpartum period and both average levels and changes in observed caregiving behavior across a mother-infant interaction, we found that mothers with poorer objective sleep continuity 
evidenced reduced ability to sustain maternal sensitivity when interacting with their infants in their home environments. Specifically, poorer actigraphy-based sleep continuity, as assessed via a composite of nighttime wake time after sleep onset (WASO) and number of arousals, was associated with decreases in maternal sensitivity toward infants across a ten-minute free play interaction. Our findings suggest that, although mothers with poorer sleep continuity do not demonstrate significantly lower overall levels of sensitivity toward their infants during a free play interaction, their levels of sensitivity decline over time. While statistically significant, it is important to note that the observed effect sizes were small, suggesting that maternal sleep continuity is one factor of many that may contribute to variation in maternal sensitivity toward infants.

There are several mechanisms though which poor sleep continuity may have adverse consequences for maternal sensitivity, including deficits in executive functioning and increased negative mood. Working memory, focused attention, and inhibitory control are executive functions that are critical for interpreting environmental cues that unfold over time, as well as for managing responses to these cues (Diamond, 2013). In the context of mother-infant interactions, working memory is important for the mother's ability to maintain in mind her infant's cues as well as her goals for responding to these cues. Thus, failures in working memory could result in problems interpreting later infant cues based on memory for earlier cues and inhibiting behaviors that do not align with goals for the interaction. Indeed, problems in mothers' executive functioning are associated with less time caring for infants (Chico, Gonzalez, Ali, Steiner, \& Fleming, 2014) and reduced maternal sensitivity in free-play interactions (Sturge-Apple et al., 2017). Further, mothers with chronic attention problems (i.e., ADHD) evidence more problematic patterns of parenting than mothers without these problems (for a review, see Crandall, DeaterDeckard, \& Riley, 2015).Therefore, it is possible that deficits in executive function due to poor sleep continuity (Insana et al., 2013; Wilckens, Woo, Kirk, Erickson, \& Wheeler, 2014) are a 
mechanism for the association between poorer sleep continuity and decrements in maternal sensitivity across interactions with infants.

An additional possible mechanism for the association between poorer sleep continuity and the ability to sustain sensitivity involves the effects of sleep disturbance on mothers' mood. Greater sleep disturbance has been consistently associated with more negative mood (Franzen et al., 2008; Gordon \& Chen, 2014; Kalmbach, Arnedt, Swanson, Rapier, \& Ciesla, 2017); in fact, sleep problems are a symptom of disorders defined by chronic negative mood (e.g., depression; Baglioni et al., 2016). In a longitudinal study beginning in the third trimester of pregnancy and continuing until 14 weeks postpartum, poorer maternal sleep continuity, but not sleep duration, was associated with postpartum depressive symptoms (Park et al., 2013). In the context of research on the correlates of caregiving, considerable research has documented associations between more negative mood in mothers and lower quality of caregiving behavior (Field, 2010; Lovejoy, Graczyk, O’Hare, \& Neuman, 2000). In the current study, however, we found that poorer objective sleep continuity was associated with decreases in maternal sensitivity during mother-infant interactions even when adjusting for maternal depressive symptoms. Although this finding suggests that maternal depressive symptoms do not mediate the association between poorer sleep continuity and maternal sensitivity, depressive symptoms were generally low in the current sample, likely because women with current major depression during pregnancy at the time of recruitment were excluded from the parent study. Further, it is possible that more negative in-the-moment affect, which was not measured in the current study, explains the observed association between poorer objective sleep continuity and the diminished ability to sustain maternal sensitivity when interacting with infants. Future research is needed to test this possibility.

In the current study, neither mothers' self-reported sleep disturbance nor symptoms of insomnia were significantly associated with either overall levels of maternal sensitivity or the ability to sustain sensitivity toward infants across the interaction. Sleep is a multi-dimensional 
construct that cannot be fully characterized using a single measure (Buysse, 2014) and previous research has demonstrated that different measures of sleep (e.g., nighttime sleep duration, nighttime wakefulness, self-reported sleep quality) and different methods (i.e., subjective self-reports on sleep diaries vs. objective assessments using actigraphy) are not necessarily correlated (Landry, Best, \& Liu-Ambrose, 2015; Unruh et al., 2008). In the broader literature, researchers have reported differential associations of subjective and objective measures of sleep with psychological functioning (Jackowska, Dockray, Hendrickx, \& Steptoe, 2011; Russell, Wearden, Fairclough, Emsley, \& Kyle, 2016). In an exploratory factor analysis, we found that mothers' reports of sleep disturbance on sleep diaries and actigraphy measurements of their sleep continuity loaded onto two separate factors (i.e., subjective sleep disturbance and objective sleep continuity). Thus, it is possible that subjective and objective sleep disturbance represent unique dimensions of sleep during the postpartum period with distinct consequences for mothers' functioning, and, consequently, their behavior toward their infants. While self-report measures provide important information about perceptions of sleep disturbance, these perceptions may not reflect actual sleep characteristics; in turn, actual sleep characteristics may be more consequential for caregiving behavior than are perceptions. In fact, the only previous study that has tested the associations between both subjective and objective measure of maternal sleep and caregiving behavior similarly found that actigraphy measures of mothers' sleep disturbance, but not their self-reported sleep quality, were associated with their bedtime caregiving behavior toward their toddlers (Mcquillan et al., 2019). Nonetheless, among actigraphy measures, different indices of maternal sleep may be differentially associated with postpartum functioning. Indeed, we found that objective total sleep duration was not highly correlated with other objective measures and was not significantly associated with maternal sensitivity.

Multi-dimensional and multi-method measurement of mothers' sleep is a strength of the current study. In addition, we conducted observational assessments of maternal sensitivity, 
extending previous research of the association between maternal sleep disturbance and mothers self-reports of their feelings toward or perceptions of their infants (Tikotzky, 2016; Tikotzky et al., 2012). A novel aspect of this study is the assessment of mothers' ability to sustain sensitivity across time during mother-infant interactions in the ecologically valid setting of the dyad's home. Previous research has largely ignored the time-dependent nature of caregiving behavior. The findings of the current study suggest that sustaining sensitivity may be an important construct for future investigations both in research of maternal sleep and in the broader literature on caregiving behavior. Finally, by additionally assessing infant negative mood during the free play interaction, the current study accounted for the transactional nature of parent-child interactions, whereby children's temperaments may influence the care they receive (Klein et al., 2016). We found that poorer maternal sleep continuity was associated with decrements in maternal sensitivity above and beyond infant negative mood during the interaction.

Limitations of the current study should be considered when interpreting the findings. First, the current analyses were correlational, precluding the interpretation that poor maternal sleep continuity causes decrements in maternal sensitivity across mother-infant interactions. It is possible a that a "third variable" explains this association. For example, the observed association between mothers' sleep continuity and sustained maternal sensitivity could be explained, in part, by the effect of stress on both sleep (Lewis et al., 2013) and maternal sensitivity (Booth et al., 2018). Additional methodological limitations of the current study include the relatively small sample size and that mothers were homogenous in having experienced insomnia disorder during pregnancy. Although at 18 weeks postpartum, mothers tended to endorse low insomnia severity, we should be cautious in generalizing the findings to mothers who have never experienced insomnia or who are prone to other forms of psychopathology. Generalizability of the results is hindered by the fact that most participants were Caucasian and lived in a geographic area characterized by a high cost of living where the median income is 
$>\$ 100,000$ (https://www.census.gov). Finally, the sample size for the current study was determined by retention from the parent study and not based on an a priori power analysis.

The current study is an important first step in characterizing the relation between variation in mothers' sleep and their caregiving behavior during the postpartum period. Infancy is a period of rapid development when humans depend on their caregivers to provide the psychosocial input necessary for their healthy development. Therefore, disruptions in maternal sensitivity due to poor maternal sleep continuity in this period may have long-lasting consequences for children's psychological health. Although future research is necessary to identify the mechanisms that may explain an association between poor maternal sleep continuity and the inability to sustain sensitivity toward infants, our findings suggest that mothers' objective sleep continuity may be one factor to consider when designing interventions focused on improving the parent-child relationship and preventing adverse psychological outcomes in children. 


\section{Acknowledgements}

We thank the families who participated in this study, and Drs. Margaret T. Owen and Cynthia Frosch for their assistant in designing the dyadic assessment. Funding for this study was provided by the National Institutes of Health (NIH R01 NR013662) and the National Science Foundation (graduate research fellowship to LSK). 


\section{References}

Ainsworth, M. D. S., Blehar, M. C., Waters, E., \& Wall, S. N. (1978). Patterns of attachment: $A$ psychological study of the strange situation. Hillsdale, NJ: Erlbaum.

American Psychiatric Association. (2013). Diagnostic and Statistical Manual of Mental Disorders (5th edn.). American Psychiatric Publishing.

Baglioni, C., Nanovska, S., Regen, W., Spiegelhalder, K., Feige, B., Nissen, C., ... Riemann, D. (2016). Sleep and mental disorders: A meta-analysis of polysomnographic research.

Psychological Bulletin, 142(9), 969-990. https://doi.org/10.1037/bul0000053

Bagner, D. M., Pettit, J. W., Lewinsohn, P. M., \& Seeley, J. R. (2010). Effect of maternal depression on child behavior: A sensitive period? Journal of the American Academy of Child and Adolescent Psychiatry, 49(7), 699-707. https://doi.org/10.1016/j.jaac.2010.03.012

Bastien, Â. H., Vallie, A., \& Morin, C. M. (2001). Validation of the Insomnia Severity Index as an outcome measure for insomnia research. Sleep Medicine, 2, 297-307.

Bates, D., Machler, M., Bolker, B., \& Walker, S. (2014). Fitting linear mixed-effects models using Ime4. Journal of Statistical Software, 1-51.

Belsky, J., Pasco Fearon, R. M., \& Bell, B. (2007). Parenting, attention and externalizing problems: Testing mediation longitudinally, repeatedly and reciprocally. Journal of Child Psychology and Psychiatry and Allied Disciplines, 48(12), 1233-1242. https://doi.org/10.1111/j.1469-7610.2007.01807.x

Bernard, K., Meade, E. B., \& Dozier, M. (2013). Parental synchrony and nurturance as targets in an attachment based intervention: Building upon mary ainsworth's insights about motherinfant interaction. Attachment \& Human Development, 15(5-6), 507-523. https://doi.org/10.1080/14616734.2013.820920.Parental

Bigelow, A. E., MacLean, K., Proctor, J., Myatt, T., Gillis, R., \& Power, M. (2010). Maternal sensitivity throughout infancy: Continuity and relation to attachment security. Infant 
Behavior and Development, 33(1), 50-60. https://doi.org/10.1016/j.infbeh.2009.10.009

Booth, A. T., Macdonald, J. A., \& Youssef, G. J. (2018). Contextual stress and maternal sensitivity: A meta-analytic review of stress associations with the Maternal Behavior Q-Sort in observational studies. Developmental Review, (December 2017), 0-1. https://doi.org/10.1016/j.dr.2018.02.002

Bosquet Enlow, M., Carter, A., Hails, K., King, L., \& Cabrera, I. (2014). Parent-Child Interaction Rating Scales - Infant Adaptation Manual.

Bosquet Enlow, M., King, L., Schreier, H. M., Howard, J. M., Rosenfield, D., Ritz, T., \& Wright, R. J. (2014). Maternal sensitivity and infant autonomic and endocrine stress responses. Early Human Development, 90(7), 377-385. https://doi.org/10.1016/j.earlhumdev.2014.04.007

Braungart-Rieker, J. M., Hill-Soderlund, A. L., \& Karrass, J. (2010). Fear and anger reactivity trajectories from 4 to 16 months: The roles of temperament, regulation, and maternal sensitivity. Developmental Psychology, 46(4), 791-804. https://doi.org/10.1037/a0019673

Buysse, D. J. (2014). Sleep health: Can we define it? Does it matter? Sleep, 37(1), 9-17. https://doi.org/10.5665/sleep.3298

Carney, C. E., Buysse, D. J., Ancoli-israel, S., Edinger, J. D., Krystal, A. D., Lichstein, K. L., \& Morin, C. M. (2012). The consensus sleep diary : Standardizing prospective sleep selfmonitoring. SLEEP, 35(2), 287-302.

Chico, E., Gonzalez, A., Ali, N., Steiner, M., \& Fleming, A. S. (2014). Executive function and mothering: Challenges faced by teenage mothers. Developmental Psychobiology, 56(5), 1027-1035. https://doi.org/10.1002/dev.21185

Conradt, E., \& Ablow, J. (2010). Infant physiological response to the still-face paradigm: Contributions of maternal sensitivity and infants' early regulatory behavior. Infant Behavior and Development, 33(3), 251-265. https://doi.org/10.1016/j.infbeh.2010.01.001

Costello, A. B., \& Osborne, J. W. (2005). Best practices in exploratory factor analysis : Four 
recommendations for getting the most from your analysis. Practical Assessment, Research \& Evaluation, 10(7), 1-9.

Cox, J. L., Holden, J. M., \& Sagovsky, R. (1987). Detection of postnatal depression. Development of the 10-item Edinburgh Postnatal Depression Scale. The British Journal of Psychiatry: The Journal of Mental Science, 150, 782-786.

Crandall, A. A., Deater-Deckard, K., \& Riley, A. W. (2015). Maternal emotion and cognitive control capacities and parenting: A conceptual framework. Developmental Review, 36, 105-126. https://doi.org/10.1016/j.dr.2015.01.004

Davidov, M., \& Grusec, J. E. (2006). Untangling the links of parental responsiveness to distress and warmth to child outcomes. Child Development, 77(1), 44-58.

Di Blasio, P., Camisasca, E., \& Miragoli, S. (2018). Childbirth related post-traumatic stress symptoms and maternal sleep difficulties: Associations with parenting stress. Frontiers in Psychology, 9(November), 1-12. https://doi.org/10.3389/fpsyg.2018.02103

Diamond, A. (2013). Executive functions. Annual Review of Psychology, 64, 135-168. https://doi.org/10.1146/annurev-psych-113011-143750

Field, T. (2010). Postpartum depression effects on early interactions, parenting, and safety practices: A review. Infant Behavior and Development. https://doi.org/10.1016/j.infbeh.2009.10.005

Floam, S., Simpson, N., Nemeth, E., Scott-Sutherland, J., Gautam, S., \& Haack, M. (2015). Sleep characteristics as predictor variables of stress systems markers in insomnia disorder. Journal of Sleep Research, 24(3), 296-304. https://doi.org/10.1111/jsr.12259

Franzen, P. L., Siegle, G. J., \& Buysse, D. J. (2008). Relationships between affect, vigilance, and sleepiness following sleep deprivation. Journal of Sleep Research, 17(1), 34-41. https://doi.org/10.1111/j.1365-2869.2008.00635.x

Gay, C. L., Lee, K. A., \& Lee, S.-Y. (2004). Sleep patterns and fatigue in new mothers and fathers. Biological Research For Nursing, 5(4), 311-318. 
https://doi.org/10.1177/1099800403262142

Gordon, A. M., \& Chen, S. (2014). The role of sleep in interpersonal conflict: Do sleepless nights mean worse fights? Social Psychological and Personality Science, 5(2), 168-175. https://doi.org/10.1177/1948550613488952

Holden, G. W., Williamson, P. A., \& Holland, G. W. O. (2014). Eavesdropping on the family: A pilot investigation of corporal punishment in the home. Journal of Family Psychology, 28(3), 401-406. https://doi.org/10.1037/a0036370

Hunter, L. P., Rychnovsky, J. D., \& Yount, S. M. (2009). A selective review of maternal sleep characteristics in the postpartum period. JOGNN - Journal of Obstetric, Gynecologic, and Neonatal Nursing, 38(1), 60-68. https://doi.org/10.1111/j.1552-6909.2008.00309.x

Hysing, M., Harvey, A. G., Torgersen, L., Ystrom, E., Reichborn-Kjennerud, T., \& Sivertsen, B. (2014). Trajectories and predictors of nocturnal awakenings and sleep duration in infants. Journal of Developmental and Behavioral Pediatrics, 35(5), 309-316. https://doi.org/10.1097/DBP.0000000000000064

Insana, S. P., Williams, K. B., \& Montgomery-Downs, H. E. (2013). Sleep disturbance and neurobehavioral performance among postpartum women. Sleep, 36(1), 73-81. https://doi.org/10.5665/sleep.2304

Jackowska, M., Dockray, S., Hendrickx, H., \& Steptoe, A. (2011). Psychosocial factors and sleep efficiency: Discrepancies between subjective and objective evaluations of sleep. Psychosomatic Medicine, 73(9), 810-816. https://doi.org/10.1097/PSY.0b013e3182359e77

Kalmbach, D. A., Arnedt, J. T., Swanson, L. M., Rapier, J. L., \& Ciesla, J. A. (2017). Reciprocal dynamics between self-rated sleep and symptoms of depression and anxiety in young adult women: a 14-day diary study. Sleep Medicine, 33, 6-12. https://doi.org/10.1016/j.sleep.2016.03.014

Kim, B. R., \& Teti, D. M. (2014). Maternal emotional availability during infant bedtime: An ecological framework. Journal of Family Psychology, 28(1), 1-11. 
https://doi.org/10.1037/a0035157

Klein, M. R., Lengua, L. J., Thompson, S. F., Moran, L., Ruberry, E. J., Kiff, C., \& Zalewski, M. (2016). Bidirectional relations between temperament and parenting predicting preschoolage children's adjustment. Journal of Clinical Child and Adolescent Psychology, 1-14. https://doi.org/10.1080/15374416.2016.1169537

Kok, R., Linting, M., Bakermans-Kranenburg, M. J., van ljzendoorn, M. H., Jaddoe, V. W. V., Hofman, A., ... Tiemeier, H. (2013). Maternal sensitivity and internalizing problems: Evidence from two longitudinal studies in early childhood. Child Psychiatry and Human Development, 44(6), 751-765. https://doi.org/10.1007/s10578-013-0369-7

Kraemer, H. C., Ph, D., Stice, E., Kazdin, A., Offord, D., \& Kupfer, D. (2001). How do risk factors work together? Mediators, moderators, and independent, overlapping, and proxy risk factors. Psychiatry Interpersonal and Biological Processes, 158(June), 848-856. https://doi.org/10.1176/appi.ajp.158.6.848

Kuznetsova, A., Brockhoff, P. B., \& Christensen, R. H. B. (2016). Tests in linear mixed effects models. Journal of Stastical Software, 82(13), 1-26. https://doi.org/10.18637/jss.v082.i13 Landry, G. J., Best, J. R., \& Liu-Ambrose, T. (2015). Measuring sleep quality in older adults: A comparison using subjective and objective methods. Frontiers in Aging Neuroscience, 7(SEP), 1-10. https://doi.org/10.3389/fnagi.2015.00166

Lee, R., Skinner, A., Bornstein, M. H., Radford, A. N., Campbell, A., Graham, K., \& Pearson, R. M. (2017). Through babies' eyes: Practical and theoretical considerations of using wearable technology to measure parent-infant behaviour from the mothers' and infants' view points. Infant Behavior and Development, 47, 62-71. https://doi.org/10.1016/j.infbeh.2017.02.006

Leerkes, E. M. (2011). Predictors of maternal sensitivity to infant distress. Parenting: Science and Practice, 10(3), 219-239. https://doi.org/10.1080/15295190903290840

Lewis, T. T., Troxel, W. M., Kravitz, H. M., Bromberger, J. T., Matthews, K. A., \& Hall, M. H. 
(2013). Chronic exposure to everyday discrimination and sleep in a multiethnic sample of middle-aged women. Health Psychology, 32(7), 810-819. https://doi.org/10.1037/a0029938

Long, J. A. (2018). jtools: Analysis and presentation of social scientific data. Retrieved from https://cran.r-project.org/package=jtools

Lovejoy, M. C., Graczyk, P. A., O’Hare, E., \& Neuman, G. (2000). Maternal depression and parenting behavior: A meta-analytic review. Clinical Psychology Review, 20(5), 561-592.

Manber, R., Bei, B., Simpson, N., Asarnow, L., Rangel, E., Sit, A., \& Lyell, D. (2019). Cognitive behavioral therapy for prenatal insomnia: A randomized controlled trial. Journal of Obstetrics and Gynecology.

Marino, M., Li, Y., Rueschman, M. N., Winkelman, J. W., Ellenbogen, J. M., Solet, J. M., ... Buxton, O. M. (2013). Measuring sleep: Accuracy, sensitivity, and specificity of wrist actigraphy compared to polysomnography. Sleep, 36(11), 1747-1755. https://doi.org/10.5665/sleep.3142

Mauss, I. B., Troy, A. S., \& LeBourgeois, M. K. (2013). Poorer sleep quality is associated with lower emotion-regulation ability in a laboratory paradigm. Cognition and Emotion, 27(3), 567-576. https://doi.org/10.1080/02699931.2012.727783

Mcbean, A. L., Kinsey, S. G., Montgomery-downs, H. E., \& Virginia, W. (2016). Effects of a single night of postpartum sleep on childless women's daytime functioning. Physiology and Behavior, 15(156), 137-147. https://doi.org/10.1016/j.physbeh.2016.01.014.Effects

McBean, A. L., \& Montgomery-Downs, H. E. (2013). Timing and variability of postpartum sleep in relation to daytime performance. Physiology and Behavior, 122, 134-139. https://doi.org/10.1016/j.physbeh.2013.09.003

McCambridge, J., de Bruin, M., \& Witton, J. (2012). The effects of demand characteristics on research participant behaviours in non-laboratory settings: A systematic review. PLoS ONE, 7(6), 1-6. https://doi.org/10.1371/journal.pone.0039116 
McLaughlin, K. A., Sheridan, M. A., Tibu, F., Fox, N. A., Zeanah, C. H., \& Nelson, C. A. (2015). Causal effects of the early caregiving environment on development of stress response systems in children. Proceedings of the National Academy of Sciences, 112(18), 56375642. https://doi.org/10.1073/pnas. 1423363112

Mcquillan, M. E., Bates, J. E., Staples, A. D., \& Deater-Deckard, K. (2019). Maternal stress, sleep, and parenting. Journal of Family Psychology, 33(3), 349-359. https://doi.org/10.1037/fam0000516.supp

Mezick, E. (2013). Sleep continuity. In Encyclopedia of Behavioral Medicine (pp. 1805-1806). New York, NY: Springer New York. https://doi.org/10.1007/978-1-4419-1005-9_843

Mindell, J. A., Sadeh, A., Kwon, R., \& Goh, D. Y. T. (2013). Cross-cultural comparison of maternal sleep. Sleep, 36(11), 1699-1706. https://doi.org/10.5665/sleep.3132

Montgomery-Downs, H. E., Insana, S. P., Clegg-Kraynok, M. M., \& Mancini, L. M. (2010). Normative longitudinal maternal sleep: The first 4 postpartum months. American Journal of Obstetrics and Gynecology, 203(5), 465.e1-465.e7. https://doi.org/10.1016/j.ajog.2010.06.057

Muzik, M., Bocknek, E. L., Broderick, A., Richardson, P., Rosenblum, K. L., Thelen, K., \& Seng, J. S. (2013). Mother-infant bonding impairment across the first 6 months postpartum: The primacy of psychopathology in women with childhood abuse and neglect histories. Archives of Women's Mental Health, 16(1), 29-38. https://doi.org/10.1007/s00737-0120312-0

Park, E. M., Meltzer-Brody, S., \& Stickgold, R. (2013). Poor sleep maintenance and subjective sleep quality are associated with postpartum maternal depression symptom severity. Archives of Women's Mental Health, 16(6), 539-547. https://doi.org/10.1007/s00737-0130356-9

Perry, N. B., Dollar, J. M., Calkins, S. D., \& Bell, M. A. (2018). Developmental cascade and transactional associations among niological and behavioral indicators of temperament and 
maternal behavior. Child Development, 89(5), 1735-1751.

https://doi.org/10.1111/cdev.12842

Perry, R. E., Blair, C., \& Sullivan, R. M. (2017). Neurobiology of infant attachment: Attachment despite adversity and parental programming of emotionality. Current Opinion in Psychology, 17, 1-6. https://doi.org/10.1016/j.copsyc.2017.04.022

Piteo, A. M., Roberts, R. M., Nettelbeck, T., Burns, N., Lushington, K., Martin, A. J., \& Kennedy, J. D. (2013). Postnatal depression mediates the relationship between infant and maternal sleep disruption and family dysfunction. Early Human Development, 89(2), 69-74. https://doi.org/10.1016/j.earlhumdev.2012.07.017

Price, A. M. H., Brown, J. E., Bittman, M., Wake, M., Quach, J., \& Hiscock, H. (2014). Children's sleep patterns from 0 to 9 years: Australian population longitudinal study. Archives of Disease in Childhood, 99(2), 119-125. https://doi.org/10.1136/archdischild-2013-304150

Revelle, W. (2018). psych: Procedures for personality and psychological Research. Evanston, Illinois: Northwestern University.

Russell, C., Wearden, A. J., Fairclough, G., Emsley, R. A., \& Kyle, S. D. (2016). Subjective but not actigraphy-defined sleep predicts next-day fatigue in chronic fatigue syndrome: $A$ prospective daily diary study. Sleep, 39(4), 937-944. https://doi.org/10.5665/sleep.5658

Sinai, D., \& Tikotzky, L. (2012). Infant sleep, parental sleep and parenting stress in families of mothers on maternity leave and in families of working mothers. Infant Behavior and Development, 35(2), 179-186. https://doi.org/10.1016/j.infbeh.2012.01.006

Slama, H., Chylinski, D. O., Deliens, G., Leproult, R., Schmitz, R., \& Peigneux, P. (2017). Sleep deprivation triggers cognitive control impairments in task-goal switching. Sleep, (0161-8105 (Linking)). https://doi.org/10.1093/sleep/zsx200/4737239

Smyke, A. T., Zeanah, C. H., Fox, N. A., Nelson, C. A., \& Guthrie, D. (2010). Placement in foster care enhances quality of attachment among young institutionalized children. Child Development, 81(1), 212-223. 
Sosinsky, L. S., Marakovitz, S., \& Carter, A. S. (2004). Parent-Child Interaction Rating Scales (PCIRS). Unpublished Manual. University of Massachusetts Boston. JOUR.

Sturge-Apple, M. L., Jones, H. R., \& Suor, J. H. (2017). When stress gets into your head:

Socioeconomic risk, executive functions, and maternal sensitivity across childrearing contexts. Journal of Family Psychology, 31(2), 160-169.

https://doi.org/10.1037/fam0000265

Swain, A. M., O’Hara, M. W., Starr, K. R., \& Gorman, L. L. (1997). A prospective study of sleep, mood, and cognitive function in postpartum and nonpostpartum women. Obstetrics and Gynecology, 90(3), 381-386. Retrieved from http://www.ncbi.nlm.nih.gov/pubmed/9277648

Team, R. C. (2018). R: A language and environment for statistical computing.

Tester-Jones, M., Karl, A., Watkins, E., \& O’Mahen, H. (2016). Rumination in dysphoric mothers negatively affects mother-infant interactions. Journal of Child Psychology and Psychiatry. https://doi.org/10.1111/jcpp.12633

Thomas, J. C., Letourneau, N., Campbell, T. S., Tomfohr-Madsen, L., Giesbrecht, G. F., Kaplan, B. J., ... Singhal, N. (2017). Developmental origins of infant emotion regulation: Mediation by temperamental negativity and moderation by maternal sensitivity. Developmental Psychology, 53(4), 611-628. https://doi.org/10.1037/dev0000279

Thomson, D. R., Besner, D., \& Smilek, D. (2015). A resource-control account of sustained attention: Evidence from mind-wandering and vigilance paradigms. Perspectives on Psychological Science, 10(1), 82-96. https://doi.org/10.1177/1745691614556681

Tikotzky, L. (2016). Postpartum maternal sleep, maternal depressive symptoms and selfperceived mother-infant emotional relationship. Behavioral Sleep Medicine, 14(1), 5-22. https://doi.org/10.1080/15402002.2014.940111

Tikotzky, L., Chambers, A. S., Gaylor, E., \& Manber, R. (2010). Maternal sleep and depressive symptoms: Links with infant Negative Affectivity. Infant Behavior and Development, 33(4), 605-612. https://doi.org/10.1016/j.infbeh.2010.07.012 
Tikotzky, L., Chambers, A. S., Kent, J., Gaylor, E., \& Manber, R. (2012). Postpartum maternal sleep and mothers' perceptions of their attachment relationship with the infant among women with a history of depression during pregnancy. International Journal of Behavioral Development, 36(6), 440-448. https://doi.org/10.1177/0165025412450528

Tikotzky, L., Sadeh, A., Volkovich, E., Manber, R., Meiri, G., \& Shahar, G. (2015). Infant sleep development from 3 to 6 months postpartum: Links with maternal sleep and paternal involvement. Monographs of the Society for Research in Child Development, 80(1), 107124. https://doi.org/10.1111/mono.12147

Unruh, M. L., Redline, S., An, M. W., Buysse, D. J., Nieto, F. J., Yeh, J. L., \& Newman, A. B. (2008). Subjective and objective sleep quality and aging in the sleep heart health study. Journal of the American Geriatrics Society, 56(7), 1218-1227. https://doi.org/10.1111/j.1532-5415.2008.01755.x

Volkovich, E., Bar-Kalifa, E., Meiri, G., \& Tikotzky, L. (2017). Mother-infant sleep patterns and parental functioning of room-sharing and solitary sleeping families: a longitudinal study from 3 to 18 months. Sleep, (March), 1-14. https://doi.org/10.1093/sleep/zsx207

Whitney, P., Hinson, J. M., Satterfield, B. C., Grant, D. A., Honn, K. A., \& Van Dongen, H. P. A. (2017). Sleep deprivation diminishes attentional control effectiveness and impairs flexible adaptation to changing conditions. Scientific Reports, 7(1), 1-9. https://doi.org/10.1038/s41598-017-16165-z

Wilckens, K. A., Woo, S. G., Kirk, A. R., Erickson, K. I., \& Wheeler, M. E. (2014). Role of sleep continuity and total sleep time in executive function across the adult lifespan. Psychology and Aging, 29(3), 658-665. https://doi.org/10.1037/a0037234

Wolff, M., \& van IJzendoorn, M. H. (1997). Sensitivity and attachment: A meta-analysis on parental antecedents of infant attachment. Child Development, 68(4), 571-591.

Woltman, H., Feldstain, A., Mackay, J. C., \& Rocchi, M. (2012). An introduction to hierarchical linear modeling. Tutorials in Quantitative Methods for Psychology, 8(1), 52-69. 